\title{
Effect of Combined Physical and Cognitive Interventions on Executive Functions in Older Adults: A Meta-Analysis of Outcomes
}

\author{
Wei Guo ${ }^{1,2}$, Ming Zang ${ }^{1}$, Sebastian Klich ${ }^{3}{ }^{\mathbb{D}}$, Adam Kawczyński ${ }^{3}$, \\ Małgorzata Smoter ${ }^{4}$ and Biye Wang $1,2, * \mathbb{D}$ \\ 1 College of Physical Education, Yangzhou University, Yangzhou 225009, China; guowei@yzu.edu.cn (W.G.); \\ zangming@yzu.edu.cn (M.Z.) \\ 2 Institute of Sports, Exercise and Brain, Yangzhou University, Yangzhou 225009, China \\ 3 Department of Paralympic Sport, University School of Physical Education in Wrocław, \\ 51-617 Wrocław, Poland; sebastian.klich@awf.wroc.pl (S.K.); adam.kawczynski@awf.wroc.pl (A.K.) \\ 4 Diagnostic and Rehabilitation Center 'Promyk Słońca', 50-088 Wrocław, Poland; m.h.smoter@gmail.com \\ * Correspondence: wangbiye@yzu.edu.cn
}

Received: 27 July 2020; Accepted: 23 August 2020; Published: 25 August 2020

\begin{abstract}
Background: Both physical exercise and cognitive training can effectively improve executive functions in older adults. However, whether physical activity combined with cognitive training is more effective than a single intervention remains controversial. The aim of this study was to perform a meta-analysis to evaluate the effect of combined physical and cognitive interventions on executive functions in older adults aged 65-80 years old. Methods: Randomized controlled trials of combined physical and cognitive interventions on executive functions in older adults were searched using the Web of Science, Elsevier Science, PubMed, EBSCO, Springer-Link, and NATURE databases. Data extraction and quality evaluation were done by Comprehensive Meta-Analysis, V3. Results: A total of 21 studies were included. The results showed that the combined physical and cognitive interventions produced significantly larger gains in executive functions, compared to the control group (standardized mean difference (SMD) $=0.26,95 \%$ confidence interval (CI) $[0.14,0.39], p<0.01)$. Furthermore, the effects of the combined physical and cognitive interventions were moderated by the study quality, intervention length, and intervention frequency. No significant differences were found between the combined interventions and the physical intervention alone $(\mathrm{SMD}=0.13,95 \% \mathrm{CI}[-0.07,0.33], p>0.05)$ or the cognitive intervention alone $(\mathrm{SMD}=0.13,95 \% \mathrm{CI}$ $[-0.05,0.30], p>0.05)$. Conclusions: The combined physical and cognitive interventions effectively delayed the decrease of executive functions in older adults and this effect was influenced by the length and frequency of the intervention as well as the research quality. However, the effect of the combined physical and cognitive interventions was not significantly better than that of each intervention alone.
\end{abstract}

Keywords: combined physical and cognition interventions; executive functions; meta-analysis; older adults

\section{Introduction}

Executive functions (EFs), also known as executive control or cognitive control, refers to a set of top-down mental processes [1]. The framework of EFs, which is divided into response inhibition, complex EFs, set-shifting, and updating, has been widely accepted [2-5]. It has been proven that the decline of EFs in older adults can seriously affect their daily lives and is the main cause of cognitive aging [6,7]. Therefore, it is important to counteract the decline of EFs in older adults. Fortunately, some methods have been found to delay this process. Physical activities are recommended as an effective nonpharmacological approach to improve EFs in healthy older adults [8-11]. In 
addition, a recent meta-analysis has reported that physical activities can effectively improve the EFs of older adults with mild cognitive impairment [12]. Meanwhile, cognitive training is another type of behavioral intervention that has been demonstrated to effectively delay cognitive aging. Examples of cognitive training include playing video games [13,14], television-based cognitive training [15], computerized cognitive training [16,17], playing board games [18,19], and mathematical training [20]. In terms of the efficacy of cognitive training in older adults with mild cognitive impairment, studies published over the past decade have revealed that cognitive training can effectively enhance specific cognitive functions, such as attention, orientation, verbal fluency, visual memory, etc. [21-23].

Based on the effect and mechanism revealed by previous studies, it is assumed that the combination of cognitive training and physical exercise may bring greater cognitive benefits than physical exercise or cognitive training alone [24-27]. Physical exercise can enhance cell proliferation and synaptic plasticity and cognitive training can guide and integrate new neurons and synapses into existing neural networks; therefore, the combination of the two interventions may induce superimposed cognitive benefits $[28,29]$. However, whether such a benefit exists remains controversial in older adults with or without cognitive impairment $[26,27,30]$. A systematic review has revealed an additional cognitive benefit of the combined interventions in healthy older adults but not in older adults with cognitive impairment [31]. In contrast, recent randomized controlled trials have shown that the combination of physical exercise and cognitive training can also improve cognitive functions in older adults with cognitive impairment [32,33].

Most of the studies mentioned above focused on the beneficial effects of physical exercise and cognitive training on overall cognitive functions, while only a few studies focused on specific cognitive functions, especially EFs. Thus, the main purpose of the present study was to explore whether the combination of cognitive training and physical exercise can effectively delay the decline of EFs in older adults. Furthermore, the current meta-analysis focused on potential moderating variables, such as the mode of combination. Studies have shown that different modes of combination may cause different results. The separation of the combined interventions is a possible cause of the negative result [34]. Meanwhile, simultaneous combined interventions have been demonstrated to induce beneficial effects on cognitive functions in older adults $[24,35,36]$. The possible reason for this discrepancy is that neural plasticity is influenced by time factors and will return to the baseline level at one hour after exercise. Therefore, the simultaneous combined interventions could obtain more cognitive benefits before returning to baseline [37]. In addition, other crucial variables, including cognitive status, intervention length, intervention frequency, session length, and research quality, were included as moderators.

The present meta-analysis adopted Comprehensive Meta-Analysis software to quantitatively evaluate the effect of the combined interventions on EFs in older adults to compare the effects between the combined intervention group, the physical intervention alone group, the cognitive intervention alone group, and the control group. Furthermore, the moderating effects were explored for all the following variables: intervention plan, research quality, and characteristics of the sample.

\section{Materials and Methods}

\subsection{Search Strategy}

The meta-analysis was conducted in accordance with the Preferred Reporting Items for Systematic Reviews and Meta-Analyses (PRISMA) statement. Systematic computer-based searches of the Web of Science, Elsevier Science, PubMed, and NATURE databases since the start of each database until December 2019 were performed. The following keywords were used: combined intervention terms ("combined" OR "multimodal" OR "dual-task"), AND physical intervention terms ("exercise" OR "physical" OR "training"), AND cognitive intervention terms ("cognitive function" OR "mental training" OR "EFs"), AND aging population terms ("older adult" OR "aged" OR "aging"). The articles in 
the selected journals were further screened and additional searches were conducted using the same search terms in Google Scholar to identify other potentially relevant articles.

\subsection{Eligibility Criteria}

Studies were considered eligible for this meta-analysis if the following criteria were fulfilled: (1) the study design must be a randomized controlled trial. (2) The sample population must be older adults (healthy or with mild cognitive impairment), but the patients could not have any neurological conditions other than mild cognitive impairment, such as a major depression. The age range had not been pre-determined and eventually resulted from the selected articles. (3) The intervention strategies must include a combination of physical and cognitive training as well as at least one comparison group in the article. (4) The intervention must have been carried out for more than 6 weeks. (5) Enough information must be reported in the article to calculate an effect size for at least one EF outcome measure. (6) The article must be written in English. The following types of studies were excluded: (1) nonintervention studies, (2) unpublished studies, abstracts, or papers, (3) review articles or theoretical articles, (4) those with interventions that were not adequately explained, and (5) the combined intervention group was not compared with any of these following groups: an active/passive control group, a physical intervention alone group, or a cognitive intervention alone group.

\subsection{Data Extraction and Analysis}

Except for the overall EFs, the present meta-analysis also divided EFs into distinguished sub-functions based on the classification used in a recent meta-analysis [38]. The tasks we chose to tap the response inhibition were Flanker task, Stroop test, and letter sets test. All the tasks require participants to suppress an interfering dominant response in order to achieve a specific goal. Set-shifting included trail-making test A and B, switching test, Digit-Symbol Substitution Test, and Dimensional Change Card Sort Test and these tasks require participants to monitor and code the input information related to the current task and replace the old information with the new information. Complex executive function included Self-Ordered Pointing task, Matrix Reasoning Test, Complex Figure Test, Behavioral Assessment of the Dysexecutive Syndrome, Controlled Oral Words Association Test, Groton Maze Learning test, Regensburger Wort Flüssigkeits-Test, and Leistungs-Prüf-System. All of these tasks include a series of components such as planning, reasoning, and problem-solving. The reason for excluding the updating component of EFs was that there were only three studies using updating tasks (N-back task and Subtest Digit Span Backwards test) in the included literature [25,34,39]. The methodology and important trial characteristics, including the study population, intervention type, and comparison of multi-armed intervention groups, were obtained from the articles and listed in a spreadsheet.

For the meta-analysis, EFs outcome data were extracted in the form of means, standard deviations (SDs), and the number of participants of each group at baseline and post-intervention. If the means and SDs were not available, the changes in the mean and SD between, at baseline, and after the intervention were extracted, or the upper and lower limits of the $95 \%$ confidence interval (CI) were used to calculate the SDs, and Comprehensive Meta-Analysis software was used for quantitative synthesis. In this study, the combined intervention group was compared with the control group, the cognitive intervention alone group, and the physical intervention alone group, respectively, and the intervention effect was measured by the standardized mean difference (SMD) after comparison. A positive effect size indicates that the combined intervention group has a better intervention effect than the comparison group. After the effect size of each study was obtained, the combined effect size and the $95 \% \mathrm{CI}$ were calculated to evaluate the effect of the combined interventions on EFs in older adults.

\subsection{Evaluation of Methodological Quality}

The methodological quality of the identified articles was evaluated independently by two reviewers (M.Z. and B.W.). A 13-item checklist modified from the Delphi list was used to assess 
the methodological quality [40] and any disagreements on ratings were discussed with a third reviewer (W.G.) before a final decision was determined. One point was awarded for each fulfilled criterion on the scale and no points were awarded if the criterion was not met; the quality score for each article ranged from 0 to 13. A total score of 10-13 points was defined as a high methodological quality, a total score of 7-9 points was defined as a medium methodological quality, and a total score of less than 7 points suggested a low methodological quality [31].

\section{Results}

\subsection{Included Studies}

A total of 21 articles were included in this meta-analysis. A summary of the specific selection process is presented in Figure 1, according to the recommended PRISMA flow diagram.


Figure 1. Selection process of the meta-analysis.

A total of 1665 participants were included in the 21 eligible articles of this meta-analysis. Among these included studies, 14 studies focused on healthy older adults and 7 studies focused on older adults with mild cognitive impairment. All participants were older than 50 years old. The mean age of the participants ranged from 67.0 to 77.9, except for one study, which did not give an exact mean age [41]. About $69 \%$ of participants were female, excepted for 2 studies, which did not report the gender [41,42]. Sample size ranged from 10 to 261.

Nine studies adopted combined physical and cognitive interventions simultaneously and 12 studies adopted sequential interventions. The simultaneous integrated physical and cognitive interventions 
consisted of dual-tasks $(n=4)$, exergames $(n=2)$, tai chi $(n=2)$, or virtual reality $(n=1)$. In terms of comparison groups, six studies included four comparison groups, three studies included three comparison groups, and 12 studies included two comparison groups. In the studies that included a comparison to a control group, eight of the studies used an active control group design and the main forms were health education activities and static stretching. Table 1 illustrates the main characteristics of the included articles.

The study quality scores ranged from 7 to $12 ; 16$ studies were supposed to be of high quality, while five studies were considered low quality. The results of Delphi list are presented in Table 1 and Supplementary Materials Table S1

\subsection{Combined Interventions vs. the Control Group}

A total of 17 articles compared the EFs of older adults between a combined intervention group and a control group [34,41-56]. As shown in Figure 2, the effect of the combined interventions was significantly better than that of the control group (standardized mean difference (SMD) $=0.26,95 \%$ confidence interval (CI) [0.14, 0.39], $p<0.01$ ). The heterogeneity test results revealed no significant heterogeneity between the two groups $\left(\mathrm{Q}(16)=19.61, \mathrm{I}^{2}=18.42, p=0.24\right)$.

\begin{tabular}{lrrrrr} 
Study name & \multicolumn{3}{c}{ Statistics for each study } \\
\cline { 3 - 5 } & $\begin{array}{l}\text { Std diff } \\
\text { in means }\end{array}$ & $\begin{array}{c}\text { Lower } \\
\text { limit }\end{array}$ & $\begin{array}{c}\text { Upper } \\
\text { limit }\end{array}$ & p-Value \\
Hiyamizu (2012) & 0.13 & -0.53 & 0.78 & 0.71 \\
Legault (2011) & -0.03 & -0.68 & 0.62 & 0.92 \\
Shatil (2013) & 0.25 & -0.26 & 0.75 & 0.34 \\
Lam (2012) & 0.07 & -0.18 & 0.33 & 0.57 \\
Sungkarat (2017) & 0.68 & 0.16 & 1.21 & 0.01 \\
Singh (2014) & 0.08 & -0.49 & 0.65 & 0.79 \\
Park (2019) & 0.45 & -0.12 & 1.02 & 0.12 \\
Kayama (2014) & 0.49 & -0.15 & 1.14 & 0.13 \\
Schoene (2013) & 0.42 & -0.29 & 1.12 & 0.25 \\
Maillot (2012) & 1.35 & 0.55 & 2.15 & 0.00 \\
Barnes (2013) & 0.19 & -0.31 & 0.68 & 0.46 \\
Mrakic (2018) & 0.24 & -1.01 & 1.48 & 0.71 \\
Brinke (2019) & 0.61 & 0.17 & 1.05 & 0.01 \\
Shah (2014) & 0.19 & -0.25 & 0.64 & 0.40 \\
Bae (2019) & 0.10 & -0.33 & 0.53 & 0.66 \\
Nishiguchi (2015) & -0.07 & -0.64 & 0.50 & 0.81 \\
Linde (2014) & 0.64 & -0.11 & 1.39 & 0.10 \\
overall & 0.26 & 0.14 & 0.39 & 0.00
\end{tabular}

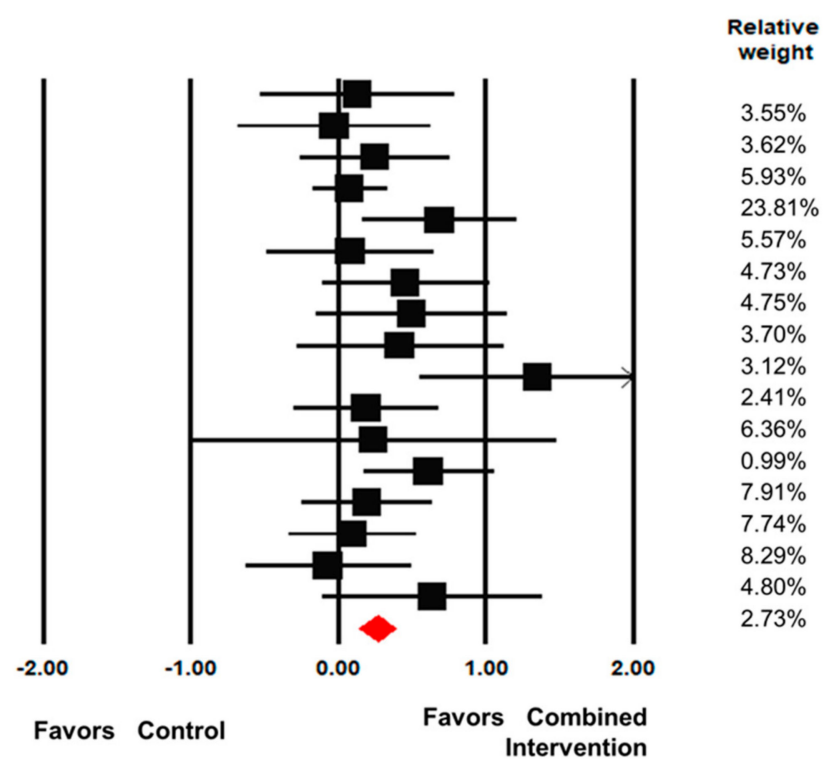

Figure 2. Forest plot for the effect sizes of the combined interventions compared to the control. 
Table 1. Main Characteristics of the Included Studies.

\begin{tabular}{|c|c|c|c|c|c|c|c|c|c|c|c|}
\hline \multicolumn{5}{|c|}{ Characteristics } & \multicolumn{4}{|c|}{ Intervention Methods } & \multirow{2}{*}{ EFs Measure Tasks } & \multirow{2}{*}{$\begin{array}{l}\text { Control Group } \\
\text { Activities }\end{array}$} & \multirow{2}{*}{ Study Quality } \\
\hline Study & Sample Size & Age (Mean) & Cognitive Status & Comparison & Cognitive Intervention & $\begin{array}{l}\text { Physical } \\
\text { Intervention }\end{array}$ & $\begin{array}{l}\text { Combination } \\
\text { Mode }\end{array}$ & Intervention Plan & & & \\
\hline Hiyamizu (2012) & 36 & $\begin{array}{l}>65 \\
(71.6)\end{array}$ & Health & CPI vs. CG & $\begin{array}{l}\text { memory, visual search, } \\
\text { verbal fluency training }\end{array}$ & Balance exercise & Simultaneous & $\begin{array}{c}60 \mathrm{~min} / \mathrm{session}, \\
2 \text { sessions/week, } \\
12 \text { weeks }\end{array}$ & $\begin{array}{c}\text { Stroop Task, TMT A, } \\
\text { TMT B }\end{array}$ & NO & 8 \\
\hline Legault (2011) & 73 & $\begin{array}{l}70-85 \\
(76.4)\end{array}$ & Health & CPI vs. PI vs. CI vs. CG & Memory training & $\begin{array}{l}\text { Aerobic } \\
\text { and flexibility exercises }\end{array}$ & Separate & $\begin{array}{c}50-150 \mathrm{~min} / \mathrm{session} \\
3 \text { sessions/week, } \\
24 \text { weeks }\end{array}$ & $\begin{array}{l}\text { 2-Back Test, Flanker } \\
\text { Task, Task Switching, } \\
\text { TMT A, TMT B, } \\
\text { Self-Ordered } \\
\text { Pointing Task } \\
\end{array}$ & NO & 9 \\
\hline Maillot (2012) & 30 & $\begin{array}{l}65-78 \\
(73.5)\end{array}$ & Health & CPI vs. CG & Nintendo Wii game & $\begin{array}{l}\text { Wii Sports, Wii Fit, } \\
\text { Mario \& Sonic on } \\
\text { Olympic Games. }\end{array}$ & Simultaneous & $\begin{array}{l}60 \mathrm{~min} / \text { session, } \\
2 \text { sessions/week, } \\
12 \text { weeks }\end{array}$ & $\begin{array}{l}\text { TMT A, TMT B; } \\
\text { Stroop Test, Letter } \\
\text { Sets Test, Matrix } \\
\text { Reasoning Test, } \\
\text { Digit-Symbol } \\
\text { Substitution }\end{array}$ & NO & 9 \\
\hline Barnes (2013) & 126 & $\begin{array}{l}\geq 65 \\
(73.4)\end{array}$ & Health & CPI vs. CI vs. PI vs. CG & $\begin{array}{c}\text { Visual and } \\
\text { auditory processing } \\
\text { speed training } \\
\end{array}$ & Aerobic exercise & Separate & $\begin{array}{l}60 \mathrm{~min} / \mathrm{session}, \\
6 \text { sessions/week, } \\
12 \text { weeks }\end{array}$ & $\begin{array}{l}\text { TMT B, Erikson } \\
\text { Flanker Test }\end{array}$ & $\begin{array}{l}\text { Health video } \\
\text { education activities }\end{array}$ & 11 \\
\hline Shatil (2013) & 122 & $\begin{array}{l}65-93 \\
(76.8)\end{array}$ & Health & CPI vs. CI vs. PI vs. CG & $\begin{array}{c}\text { Cognitive game } \\
\text { (Cognifit) }\end{array}$ & $\begin{array}{l}\text { Aerobic, strength, } \\
\text { and flexibility exercises }\end{array}$ & Separate & $\begin{array}{c}40-45 \mathrm{~min} / \mathrm{session}, \\
6 \text { sessions/week, } \\
16 \text { weeks }\end{array}$ & $\begin{array}{c}\text { CogniFit } \\
\text { neuropsycholog-ical } \\
\text { evaluation }\end{array}$ & Join a book club & 7 \\
\hline $\begin{array}{l}\text { Nishiguchi } \\
\text { (2015) }\end{array}$ & 48 & $\begin{array}{r}\geq 60 \\
(73.3)\end{array}$ & Health & CPI vs. CG & $\begin{array}{l}\text { verbal fluency, } \\
\text { cognitive-motor training }\end{array}$ & $\begin{array}{l}\text { Step exercises, } \\
\text { stretching, } \\
\text { and strength }\end{array}$ & Simultaneous & $\begin{array}{c}90 \mathrm{~min} / \text { /session, } \\
1 \text { session/week, } \\
12 \text { weeks } \\
\end{array}$ & TMT A, TMT B & NO & 9 \\
\hline Rahe (2015)a & 68 & $\begin{array}{l}50-85 \\
(68.4)\end{array}$ & Health & CPI vs. CI & $\begin{array}{l}\text { Working memory, } \\
\text { fluency, inhibition, } \\
\text { planning training }\end{array}$ & $\begin{array}{l}\text { Strength, flexibility, } \\
\text { coordination, } \\
\text { and endurance exercises }\end{array}$ & Separate & $\begin{array}{l}90 \mathrm{~min} / \mathrm{session}, \\
2 \text { sessions/week, } \\
7 \text { weeks }\end{array}$ & $\begin{array}{l}\text { Regensburger Wort } \\
\text { Flüssigkeits-Test, } \\
\text { Stroop Test, WAIS-II } \\
\text { (DSB) }\end{array}$ & $\mathrm{N} / \mathrm{C}$ & 8 \\
\hline Rahe (2015)b & 30 & $\begin{array}{l}50-85 \\
(66.7)\end{array}$ & Health & CPI vs. CI & $\begin{array}{l}\text { memory, attention, } \\
\text { EFs training }\end{array}$ & $\begin{array}{l}\text { Strength, flexibility, } \\
\text { and balance exercises }\end{array}$ & Separate & $\begin{array}{l}90 \mathrm{~min} / \mathrm{session}, \\
2 \text { sessions/week, } \\
7 \text { weeks }\end{array}$ & $\begin{array}{l}\text { Complex Figure Test, } \\
\text { TMT A, TMT B }\end{array}$ & $\mathrm{N} / \mathrm{C}$ & 9 \\
\hline Kalbe (2018) & 55 & $\begin{array}{l}50-85 \\
(68.1)\end{array}$ & Health & CPI vs. CI & $\begin{array}{l}\text { Memory, attention, } \\
\text { EFs training }\end{array}$ & $\begin{array}{l}\text { Strength, flexibility, } \\
\text { and balance exercises }\end{array}$ & Separate & $\begin{array}{l}90 \mathrm{~min} / \mathrm{session}, \\
2 \text { sessions/week, } \\
7 \text { weeks }\end{array}$ & $\begin{array}{c}\text { Stroop Test, WAIS-II } \\
\text { (DSB),Regensburger } \\
\text { Wort } \\
\text { Flüssigkeits-Test }\end{array}$ & $\mathrm{N} / \mathrm{C}$ & 10 \\
\hline Lam (2012) & 261 & $\begin{array}{l}\geq 65 \\
(77.8)\end{array}$ & MCI & CPI vs. CG & Memory, attention training & Tai Chi & Simultaneous & $\begin{array}{l}30 \mathrm{~min} / \mathrm{session}, \\
3 \text { sessions/week, } \\
12 \text { months }\end{array}$ & ТМт в & Stretching exercise & 9 \\
\hline Hagovska (2017) & 80 & $\begin{array}{l}\geq 65 \\
(67)\end{array}$ & MCI & CPI vs. PI & $\begin{array}{l}\text { Attention, memory, } \\
\text { EFs training }\end{array}$ & $\begin{array}{l}\text { Different forms } \\
\text { of walking }\end{array}$ & Simultaneous & $\begin{array}{l}30 \mathrm{~min} / \mathrm{session}, \\
2 \text { sessions/week, } \\
10 \text { weeks }\end{array}$ & Stroop Test, TMT A & $\mathrm{N} / \mathrm{C}$ & 10 \\
\hline Sungkarat (2017) & 66 & $\begin{array}{l}\geq 60 \\
(67.9)\end{array}$ & MCI & CPI vs. CG & Memory, attention training & Tai Chi & Simultaneous & $\begin{array}{c}50 \mathrm{~min} / \mathrm{session}, \\
3 \text { sessions/week, } \\
12 \text { weeks }\end{array}$ & TMT A, TMT B & Education activities & 9 \\
\hline
\end{tabular}


Table 1. Cont.

\begin{tabular}{|c|c|c|c|c|c|c|c|c|c|c|c|}
\hline Mrakic (2018) & 10 & $\begin{array}{l}\geq 65 \\
(73.3)\end{array}$ & MCI & CPI vs. CG & $\begin{array}{l}\text { Memory, visuospatial } \\
\text { ability training }\end{array}$ & Aerobic exercise & Simultaneous & $\begin{array}{c}40-45 \mathrm{~min} / \mathrm{session}, \\
3 \text { sessions/week, } \\
6 \text { weeks }\end{array}$ & TMT A & NO & 8 \\
\hline Singh (2014) & 86 & $\begin{array}{l}55-89 \\
(70.1)\end{array}$ & MCI & CPI vs. CI vs. PI vs. CG & $\begin{array}{l}\text { Memory, attention, EFs, } \\
\text { cognitive processing } \\
\text { speed training }\end{array}$ & Resistance training & Separate & $\begin{array}{l}\text { 60-100 } \mathrm{min} / \mathrm{session}, \\
2 \text { sessions/week, } \\
24 \text { weeks }\end{array}$ & $\begin{array}{l}\text { WAIS-III, Controlled } \\
\text { Oral Words } \\
\text { Association Test }\end{array}$ & $\begin{array}{c}\text { Stretching } \\
\text { and education } \\
\text { activities }\end{array}$ & 12 \\
\hline Park (2019) & 49 & $\begin{array}{l}>60 \\
(71.6)\end{array}$ & MCI & CPI vs. CG & $\begin{array}{c}\text { Phrase play, } \\
\text { memory play, } \\
\text { arithmetic training }\end{array}$ & $\begin{array}{c}\text { Aerobic, } \\
\text { balance, stretching }\end{array}$ & Simultaneous & $\begin{array}{l}110 \mathrm{~min} / \mathrm{session}, \\
2 \text { sessions/week, } \\
24 \text { weeks }\end{array}$ & $\begin{array}{l}\text { Symbol-Digit } \\
\text { Substitution Test }\end{array}$ & NO & 10 \\
\hline Kayama (2014) & 41 & $\geq 65$ & Health & CPI vs. CG & $\begin{array}{l}\text { Placement problem } \\
\text { solving (Sudoku) }\end{array}$ & Tai chi & Simultaneous & $\begin{array}{l}\text { 75-80 } \mathrm{min} / \text { session, } \\
1 \text { session/week, } \\
12 \text { weeks }\end{array}$ & TMT A, ТMT B & $\begin{array}{l}\text { Strength } \\
\text { and balance training }\end{array}$ & 7 \\
\hline Brinke (2020) & 124 & $\begin{array}{l}65-85 \\
(72.4)\end{array}$ & Health & CPI vs. CI vs. CG & $\begin{array}{l}\text { Cognitive game } \\
\text { (Fit Brains) }\end{array}$ & Brisk walking & Separate & $\begin{array}{c}60 \mathrm{~min} / \text { /session, } \\
3 \text { sessions/week, } \\
8 \text { weeks }\end{array}$ & $\begin{array}{l}\text { DCCS, Flanker Test, } \\
\text { TMT A, TMT B, } \\
\text { Stroop Test }\end{array}$ & Regular activities & 9 \\
\hline Schoene (2013) & 32 & $\begin{array}{r}\geq 65 \\
(77.9)\end{array}$ & Health & CPI vs. CG & Attention training & Step training & Simultaneous & $\begin{array}{l}15-20 \mathrm{~min} / \text { /session, } \\
\text { 2-3 sessions/week, } \\
8 \text { weeks }\end{array}$ & TMT A, TMT B & Regular activities & 10 \\
\hline Linde (2014) & 55 & $\begin{array}{l}60-75 \\
(67.1)\end{array}$ & Health & CPI vs. CI vs. PI vs. CG & $\begin{array}{c}\text { Short-term memory, } \\
\text { information processing } \\
\text { speed, } \\
\text { logical reasoning training }\end{array}$ & $\begin{array}{l}\text { Aerobic, endurance, } \\
\text { and strength training }\end{array}$ & Separate & $\begin{array}{l}\text { 60-90 min/session, } \\
2 \text { sessions/week, } \\
16 \text { weeks }\end{array}$ & Leistungs-Prüf-System & NO & 11 \\
\hline Shah (2014) & 172 & $\begin{array}{l}60-85 \\
(67.4)\end{array}$ & Health & CPI vs. CI vs. PI vs. CG & $\begin{array}{l}\text { Auditory-based BFP, } \\
\text { Visual-based IP }\end{array}$ & $\begin{array}{l}\text { Walking, } \\
\text { strength training }\end{array}$ & Separate & $\begin{array}{l}60 \mathrm{~min} / \text { /session, } \\
10 \text { sessions/week, } \\
16 \text { weeks }\end{array}$ & $\begin{array}{c}\text { Groton Maze } \\
\text { Learning, Controlled } \\
\text { Oral Word } \\
\text { Association Test } \\
\end{array}$ & NO & 9 \\
\hline Bae (2019) & 83 & $\begin{array}{l}\geq 65 \\
(75.9)\end{array}$ & MCI & CPI vs. CG & “KENKOJISEICHI" system & “KENKOJISEICHI" system & m Separate & $\begin{array}{l}90 \mathrm{~min} / \text { /session, } \\
2 \text { sessions/week, } \\
24 \text { weeks }\end{array}$ & TMT в & NO & 9 \\
\hline
\end{tabular}

MCI: mild cognitive impairment. CPI: combined cognitive and physical intervention. PI: physical intervention. CI: cognitive intervention. CG: control group. TMT A: Trail Making Test A.

TMT B: Trail Making Test B. WAIS: Wechsler Adult Intelligence Scale, DSB: Subtest Digit Span Backwards. DCCS: Dimensional Change Card Sort Test. BFP: Brain Fitness Program. IP:

Insight Program. KENKOJISEICHI: a system which includes 28 physical activities, 29 cognitive activities, and 44 social activities. N/C: No control group. 


\subsection{Combined Interventions vs. Cognitive Intervention Alone}

Ten articles reported the effects of the combined interventions and the cognitive intervention alone $[25,34,39,44,47,50,52,53,56,57]$. As shown in Figure 3, there were no significant differences between the combined interventions and the cognitive intervention alone in terms of overall EFs $(\mathrm{SMD}=0.13,95 \% \mathrm{CI}[-0.05,0.30], p=0.15)$. There was also no significant heterogeneity found in the fixed-effect model across articles $\left(\mathrm{Q}(9)=4.07, \mathrm{I}^{2}=0, p=0.91\right)$.

\begin{tabular}{lrrrrr} 
Study name & & \multicolumn{3}{c}{ Statistics for each study } & \\
\cline { 2 - 5 } & $\begin{array}{c}\text { Std diff } \\
\text { in means }\end{array}$ & $\begin{array}{c}\text { Lower } \\
\text { limit }\end{array}$ & $\begin{array}{c}\text { Upper } \\
\text { limit }\end{array}$ & p-Value \\
Legault (2011) & 0.11 & -0.54 & 0.75 & 0.75 \\
Shatil (2013) & 0.27 & -0.23 & 0.77 & 0.29 \\
Rahe (2015)a & 0.05 & -0.54 & 0.64 & 0.87 \\
Rahe (2015)b & 0.49 & -0.24 & 1.22 & 0.19 \\
Kalbe (2018) & -0.16 & -0.82 & 0.51 & 0.65 \\
Singh (2014) & -0.02 & -0.60 & 0.56 & 0.94 \\
Barnes (2013) & 0.31 & -0.19 & 0.80 & 0.23 \\
Brinke (2019) & 0.16 & -0.28 & 0.59 & 0.47 \\
Shah (2014) & -0.07 & -0.47 & 0.33 & 0.73 \\
Linde (2014) & 0.36 & -0.42 & 1.13 & 0.36 \\
overall & 0.13 & -0.05 & 0.30 & 0.15
\end{tabular}

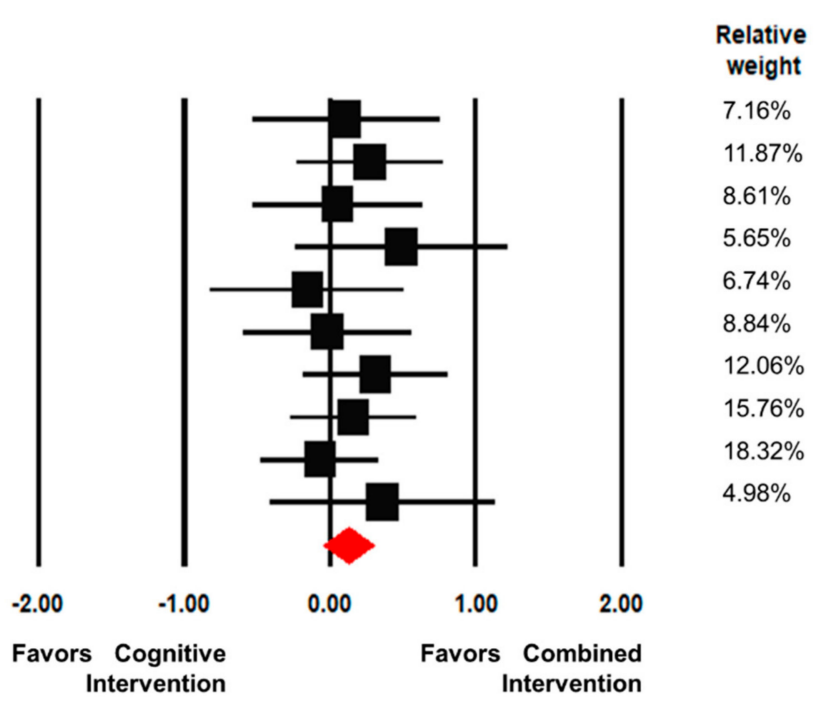

Figure 3. Forest plot for the effect sizes of the combined interventions compared to the cognitive intervention alone.

\subsection{Combined Interventions vs. Physical Exercise Intervention Alone}

Seven articles reported the effects of the combined interventions and the physical intervention alone $[34,44,47,50,53,56,58]$. As shown in Figure 4, there were no significant differences between the combined intervention groups and the physical exercise intervention alone groups (SMD $=0.13$, $95 \% \mathrm{CI}[-0.07,0.33], p=0.21)$. In addition, no significant heterogeneity across articles was found (Q (6) $\left.=3.25, \mathrm{I}^{2}=0, p=0.78\right)$.

\begin{tabular}{lrrrrr} 
Study name & \multicolumn{4}{c}{ Statistics for each study } \\
\cline { 3 - 5 } & $\begin{array}{c}\text { Std diff } \\
\text { in means }\end{array}$ & $\begin{array}{c}\text { Lower } \\
\text { limit }\end{array}$ & $\begin{array}{c}\text { Upper } \\
\text { limit }\end{array}$ & p-Value \\
Legault (2011) & 0.15 & -0.49 & 0.80 & 0.64 \\
Shatil (2013) & 0.36 & -0.15 & 0.88 & 0.17 \\
Hagovska (2017) & 0.07 & -0.37 & 0.51 & 0.76 \\
Singh (2014) & -0.32 & -0.95 & 0.32 & 0.33 \\
Barnes (2013) & 0.25 & -0.25 & 0.74 & 0.33 \\
Shah (2014) & 0.07 & -0.36 & 0.49 & 0.76 \\
Linde (2014) & 0.29 & -0.41 & 1.00 & 0.42 \\
overall & 0.13 & -0.07 & 0.33 & 0.21
\end{tabular}

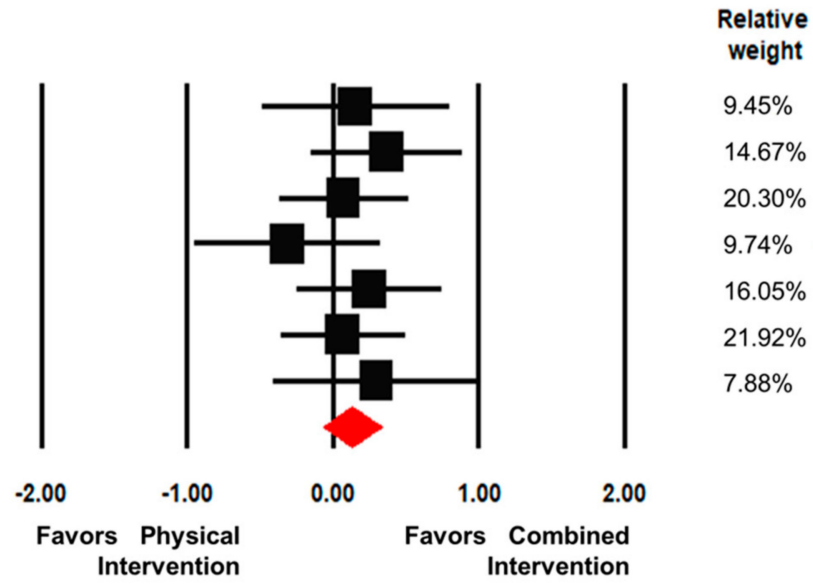

Figure 4. Forest plot for the effect sizes of the combined interventions compared to the physical intervention alone. 


\subsection{Sensitivity Analyses}

Funnel plots were used to detect a potential publication bias for the following comparisons: combined interventions vs. control, combined interventions vs. cognitive intervention alone, and combined interventions vs. physical exercise intervention alone. The results revealed that there was a risk of publication bias for the combined interventions vs. control (Figure 5), but not for the other two groups (data not shown). Sensitivity analysis was performed to identify possible outliers for the comparison between the combined interventions and the control group (mean SMD effect size $\pm 3 S D$ ). After excluding the one outlier study, there was still a significant difference in the effects between the combined intervention group and the control group (SMD $=0.23,95 \% \mathrm{CI}[0.11,0.36]$, $p<0.01)$.

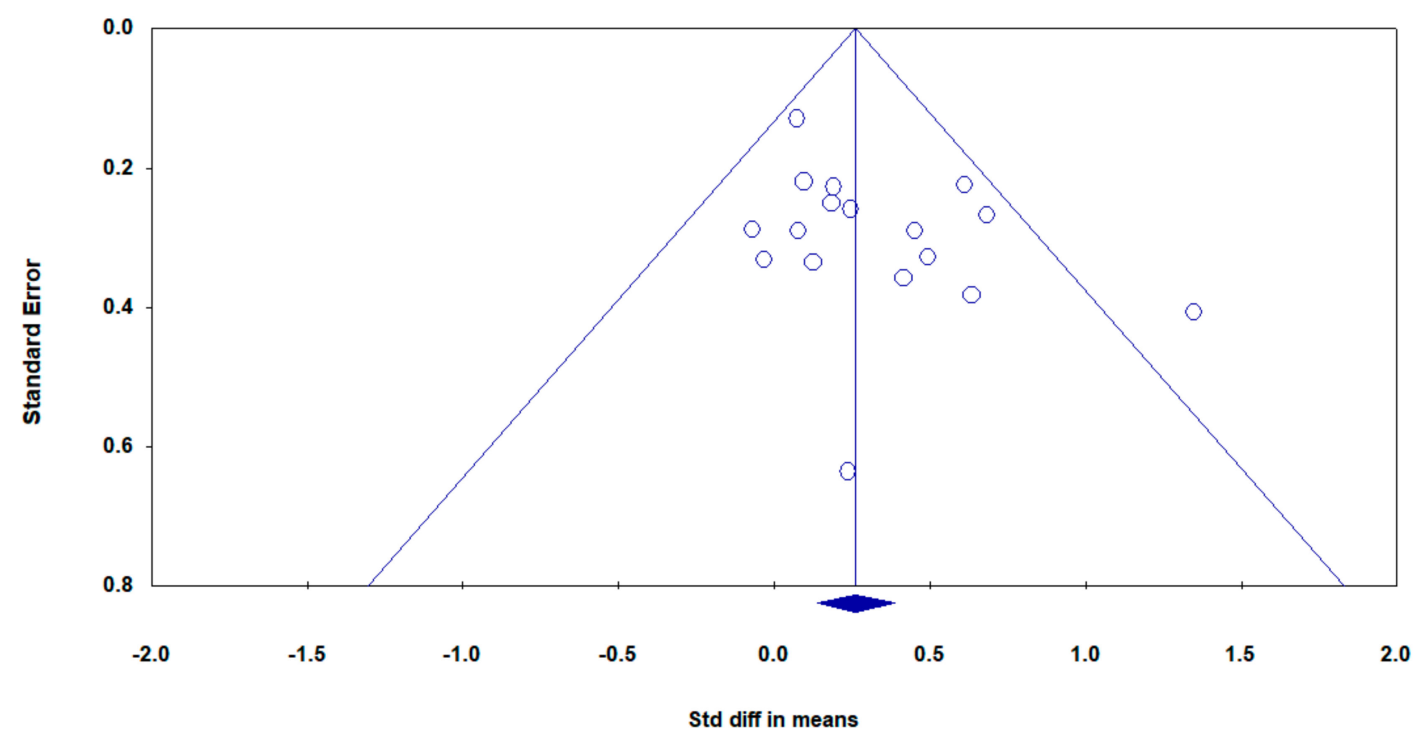

Figure 5. Funnel plot of the combined intervention group vs. the control group.

\subsection{Effect of Combined Interventions on Sub-Functions of EFs}

As shown in Table 2, compared to the control group, the combined interventions generated significant differences in response inhibition (SMD $=0.29,95 \% \mathrm{CI}[0.10,0.48], p<0.01$ ), set-shifting $(\mathrm{SMD}=0.23,95 \% \mathrm{CI}[0.10,0.37], p<0.01)$, complex EFs (SMD $=0.34,95 \%$ CI [0.13, 0.56], $p<0.01)$, and overall performance (SMD $=0.27,95 \%$ CI $[0.17,0.37], p<0.01)$. However, the results revealed significant heterogeneity in response inhibition $\left(\mathrm{Q}=20.35, p<0.01, \mathrm{I}^{2}=65.60\right)$, complex EFs $(\mathrm{Q}=19.27$, $\left.p<0.01, \mathrm{I}^{2}=63.67\right)$, and overall performance $\left(\mathrm{Q}=58.55, p<0.01, \mathrm{I}^{2}=52.18\right)$. Compared to the cognitive intervention alone, significant effects of the combined interventions were found for set-shifting (SMD $=0.28,95 \% \mathrm{CI}[0.04,0.52], p<0.01)$ and overall performance $(\mathrm{SMD}=0.13,95 \% \mathrm{CI}[0.01,0.25], p=0.03)$, but not for the other two functions. Compared to the physical intervention alone, a significant effect of the combined interventions was only observed for set shifting (SMD $=0.26,95 \% \mathrm{CI}[0.01,0.52], p=$ $0.04)$, but not for the other sub-functions. 
Table 2. Effect Sizes of the Combined Interventions on Response Inhibition, Set-Shifting, and Complex EFs.

\begin{tabular}{|c|c|c|c|c|c|c|c|c|}
\hline \multirow{2}{*}{ Comparison } & \multirow{2}{*}{ Outcomes } & \multirow{2}{*}{ No. of Studies } & \multirow{2}{*}{ SMD } & \multirow{2}{*}{ 95\% Confidence Interval } & \multirow{2}{*}{$I^{2}(\%)$} & \multicolumn{3}{|c|}{ Homogeneity Test } \\
\hline & & & & & & $\mathrm{Q}$ & df & $p$ \\
\hline \multirow{4}{*}{ Combined intervention versus Control group } & Response inhibition & 8 & $0.29^{* *}$ & 0.10 to 0.48 & 65.6 & 20.35 & 7 & 0.005 \\
\hline & Set-shifting & 13 & $0.24^{* *}$ & 0.10 to 0.37 & 34.03 & 18.19 & 12 & 0.11 \\
\hline & Complex EFs & 8 & $0.34^{* *}$ & 0.13 to 0.56 & 63.67 & 19.27 & 7 & 0.007 \\
\hline & Overall & 29 & $0.27^{* *}$ & 0.17 to 0.37 & 52.18 & 58.55 & 28 & 0.001 \\
\hline \multirow{4}{*}{ Combined intervention versus Cognitive intervention } & Response inhibition & 9 & 0.09 & -0.09 to 0.26 & 0 & 5.16 & 8 & 0.74 \\
\hline & Set-shifting & 5 & 0.04 * & 0.04 to 0.50 & 0 & 1.15 & 4 & 0.87 \\
\hline & Complex EFs & 7 & 0.07 & -0.14 to 0.28 & 0 & 2.2 & 6 & 0.9 \\
\hline & Overall & 21 & $0.13 *$ & 0.01 to 0.25 & 0 & 10.6 & 20 & 0.96 \\
\hline \multirow{4}{*}{ Combined intervention versus Physical intervention } & Response inhibition & 6 & 0.06 & -0.15 to 0.26 & 33.03 & 7.47 & 5 & 0.19 \\
\hline & Set-shifting & 4 & $0.26^{*}$ & 0.01 to 0.52 & 0 & 2.04 & 3 & 0.56 \\
\hline & Complex EFs & 5 & 0.21 & -0.04 to 0.46 & 0 & 3.56 & 4 & 0.47 \\
\hline & Overall & 15 & 0.16 & 0.02 to 0.29 & 5.43 & 14.8 & 14 & 0.39 \\
\hline
\end{tabular}




\subsection{Moderator Analyses}

To explore the impacts of potential moderator variables on EFs, the following moderator variables were selected: cognitive status of the participants, mode of combination, intervention length, intervention frequency, session length, third component, control group, and methodological quality. Because significant group differences were found only in the comparison of the combined intervention group vs. the control group, analysis of the potential moderator variables was performed only for this comparison.

Table 3 summarizes the results of the potential moderator variable analysis of the combined intervention group compared with the control group. The results showed that the effect of the combined interventions was influenced by the intervention length, which was divided into three subgroups, and the results of the heterogeneity test showed that the combined intervention effects of the three subgroups were marginally different $(p=0.09, \mathrm{Q}=4.92)$. The results showed that the combined interventions had a significant effect on the medium (SMD =0.37, 95\% CI [0.17, 0.57], $p<0.01$ ) and short $(\mathrm{SMD}=0.44,95 \% \mathrm{CI}[0.12,0.75], p<0.01)$ intervention lengths, but no difference was found for the long intervention lengths. Both the sequential (SMD $=0.27,95 \%$ CI $[0.09,0.44], p<0.01$ ) and simultaneous (SMD $=0.26,95 \% \mathrm{CI}[0.08,0.44], p<0.01$ ) combined intervention forms produced significant intervention effects compared with the control group, indicating that different combined intervention strategies may have beneficial effects.

In terms of the cognitive status, the combined interventions had a significant effect on both the healthy $(\mathrm{SMD}=0.33,95 \% \mathrm{CI}[0.16,0.50], p<0.01)$ and the mild cognitive impairment $(\mathrm{SMD}=0.19$, $95 \%$ CI [0.01, 0.37], $p=0.04$ ) populations, but a larger effect size was generated in the healthy population and the intervention effect was more significant. We also found similar results for the control group. The combined interventions had a positive effect in both the active (SMD $=0.27,95 \% \mathrm{CI}[0.11,0.43], p<$ $0.01)$ and passive $(\mathrm{SMD}=0.25,95 \% \mathrm{CI}[0.05,0.45], p=0.02)$ control groups, but the effect was more significant in the active control group. Among the moderator variables, the effect of the combined interventions was significant for only one subgroup. For instance, in terms of the frequency and session duration, the combined interventions had a significant effect on interventions with a low frequency $(\mathrm{SMD}=0.28,95 \% \mathrm{CI}[0.14,0.41], p<0.01)$ and a medium duration $(\mathrm{SMD}=0.41,95 \% \mathrm{CI}[0.22,0.61], p$ $<0.01$ ). In terms of the study quality, there was a significant cognitive gain effect with high-quality studies (SMD $=0.26,95 \%$ CI $[0.12,0.39], p<0.01)$. 
Table 3. Moderator Analysis for the Combined Intervention Group vs. the Control Group.

\begin{tabular}{|c|c|c|c|c|c|c|c|c|}
\hline \multirow{2}{*}{ Moderator } & \multirow{2}{*}{ Level } & \multirow{2}{*}{ No. of Studies } & \multirow{2}{*}{ SMD } & \multirow{2}{*}{$95 \% \mathrm{CI}$} & \multirow{2}{*}{$\mathbf{I}^{2}$} & \multicolumn{3}{|c|}{ Homogeneity Test } \\
\hline & & & & & & $\mathbf{Q}$ & df & $p$ \\
\hline \multirow{2}{*}{ Mode of combination } & Sequential & 9 & $0.27 * *$ & 0.10 to 0.44 & 0 & \multirow{2}{*}{0.01} & \multirow{2}{*}{1} & \multirow{2}{*}{0.94} \\
\hline & Simultaneous & 8 & $0.26^{* *}$ & 0.08 to 0.44 & 49.53 & & & \\
\hline \multirow{2}{*}{ Cognitive status } & Healthy & 11 & $0.33^{* *}$ & 0.16 to 0.50 & 23.28 & \multirow{2}{*}{1.22} & \multirow{2}{*}{1} & \multirow{2}{*}{0.27} \\
\hline & MCI & 6 & $0.19 *$ & 0.01 to 0.37 & 6.74 & & & \\
\hline \multirow{2}{*}{ Control group } & Active & 8 & $0.27 * *$ & 0.11 to 0.43 & 14.52 & \multirow{2}{*}{0.03} & \multirow[b]{2}{*}{1} & \multirow{2}{*}{0.86} \\
\hline & Passive & 9 & $0.25 *$ & 0.05 to 0.45 & 29.78 & & & \\
\hline \multirow{3}{*}{ Intervention length } & Long ( $\geq 24$ weeks) & 5 & 0.11 & -0.07 to 0.29 & 0 & \multirow{3}{*}{4.92} & \multirow{3}{*}{2} & \multirow{3}{*}{0.09} \\
\hline & Medium (12-23 weeks) & 8 & $0.37^{* *}$ & 0.17 to 0.56 & 39 & & & \\
\hline & Short ( $<12$ weeks) & 4 & $0.44 *$ & 0.12 to 0.75 & 0 & & & \\
\hline \multirow{2}{*}{ Frequency } & High (>3 sessions/week) & 3 & 0.21 & -0.07 to 0.48 & 0 & \multirow{2}{*}{0.2} & \multirow{2}{*}{1} & \multirow{2}{*}{0.66} \\
\hline & Low ( $\leq 3$ sessions/week) & 14 & $0.28 *$ & 0.14 to 0.41 & 32.92 & & & \\
\hline \multirow{3}{*}{ Session duration } & Long $(>60 \mathrm{~min})$ & 7 & 0.20 & -0.02 to 0.41 & 0 & \multirow{3}{*}{4.13} & \multirow{3}{*}{2} & \multirow{3}{*}{0.13} \\
\hline & Medium ( $>30$ to $\leq 60 \mathrm{~min})$ & 8 & $0.41^{* *}$ & 0.22 to 0.61 & 30.47 & & & \\
\hline & Short $(\leq 30 \mathrm{~min})$ & 2 & 0.11 & -0.13 to 0.35 & 0 & & & \\
\hline \multirow{2}{*}{ Study quality } & High ( $\geq 9$ scores) & 13 & $0.26^{* *}$ & 0.13 to 0.39 & 36.65 & \multirow{2}{*}{0.01} & \multirow{2}{*}{1} & \multirow{2}{*}{0.91} \\
\hline & Low (<9 scores) & 4 & 0.28 & -0.05 to 0.61 & 0 & & & \\
\hline
\end{tabular}




\section{Discussion}

Twenty-one studies were included in this meta-analysis to explore the effect of the combined physical and cognitive interventions on EFs in older adults. The results showed that the combined interventions produced a significantly greater effect compared with the control group in overall EFs. Furthermore, the effects of moderating variables were further explored in this meta-analysis, such as subject characteristics, intervention plan, and study quality.

The present meta-analysis showed that the combined interventions had a significant effect on the overall EFs, compared with that of the control group. While compared with the physical or cognitive intervention alone, no significant differences were found. This result is slightly different from previous meta-analyses that focused on the overall cognitive functions in older adults. They found a significant cognitive advantage of the combined interventions compared with both the control group and the exercise intervention alone group, while no significant difference was found between the combined interventions and cognitive intervention alone $[59,60]$. The different choice of outcome measures may account for the controversial findings. Our study focused on the effects of combined physical and cognitive interventions on specific EFs in older adults, rather than the overall cognitive functions as in previous studies. Considering the above differences, this study explored the effect of the combined interventions on EFs because different cognitive functions seem to be affected by different types of interventions. This is also one of the important considerations for why the sub-functions of EFs were analyzed in this study.

Although no significant effect was found between the combined and single intervention groups, the effect size reached 0.13 for both the exercise and cognitive intervention alone groups, indicating that the combined interventions still had a weak advantage over the single intervention. Of the studies analyzed, three studies $[25,47,53]$ reported a negative effect for the comparison with the cognitive group and one study [47] reported a negative effect for the comparison with the physical activity group. It is assumed that the duration and intensity of the intervention may account for the negative results. First, the intervention time was too short in these studies. The duration of the intervention moderates the effect. Walsh et al. compared long-term tai chi training with short-term tai chi training and found that short-term tai chi training did not significantly improve cognitive functions in adults [61]. Second, the high-intensity intervention may cause excessive stress and cognitive fatigue in older adults, which may affect the effect size of the intervention [62].

The combined interventions produced a significant effect on all of the sub-functions, including response inhibition, set-shifting, and complex EFs of older adults compared with the control group. The significant effect is additional evidence of lifelong plasticity of the human brain [63]. While compared with physical or cognitive intervention alone, the combined interventions only showed a significant effect on set-shifting of EFs. The ceiling effect helps to explain this result [64]. It is reasonable to assume that the sub-functions of the physical or cognitive intervention group alone probably improved to a ceiling level of the older population. Another possibility is the limitation of approaches aimed to separate cognitive sub-functions in the present meta-analysis. There has been intense theoretical debate in the past two decades about the tasks' impurity, according to which EFs are entangled with other cognitive processes, which might not be easy to distinguish in the experimental tasks [65]. Also, it has been confirmed that the sub-functions of EFs are not completely independent, however, and do seem to share some underlying commonality [4].

The results of moderator analysis showed that the larger benefit of the combined interventions relative to that of the controls depended on the intervention length. The use of a medium (12-23 weeks) or short-term $(<12$ weeks) intervention produced a significant effect, while a benefit was not found for long-term ( $\geq 24$ weeks) interventions. It seems that an intervention of less than 23 weeks can effectively improve EFs in older adults. Previous meta-analyses also have revealed that a long intervention length is not necessary for older adults to produce a better cognitive effect in physical intervention studies $[66,67]$. Of note, only five studies in the present meta-analysis adopted a long-term intervention 
plan because EFs are sensitive to environmental influences in a relatively short time [68] and long-term intervention is difficult to implement in older adults.

In practice, the determination of which type of combination to choose, simultaneous or sequential, is a controversial issue. A meta-analysis has revealed that the simultaneous combination of physical exercise and cognitive training provides significantly larger gains of cognitive functions in older adults [60]. Exercise can lead to a temporary increase in brain-derived neurotrophic factor, which will return to the baseline level after a period of time. Therefore, the use of simultaneous interventions may integrate the neurotrophic effects of exercise in a timely manner [37]. Another meta-analysis has found that both simultaneous and sequential combination interventions can improve cognitive functions in older adults [59]. Recently, a randomized control trial adopted both the simultaneous and sequential combination of stationary cycling and strategy-based memory training in older adults; the results exhibited that simultaneous training improved composite memory, while sequential training improved EFs [69]. That is to say, different types of combination intervention strategies may affect different aspects of cognition. The present meta-analysis mainly focused on EFs; both the simultaneous and sequential combination interventions can result in increased EFs compared to the controls. Our results also showed that the combined intervention strategy has significant advantages in both the active and passive control groups. In terms of the intervention frequency, a low-frequency ( $\leq 3$ sessions/week) intervention produced a better intervention effect and in terms of the session duration, a medium duration was the best ( $>30 \mathrm{~min}$ to $\leq 60 \mathrm{~min}$ ). The combined interventions had significant effects on the EFs of older adults with or without cognitive impairment. Moreover, a positive correlation between the study quality and the effect of the intervention was also found. The effect of the intervention was more significant for the studies with a higher quality.

To analyze the effect of the combined interventions on EFs in older adults, the present meta-analysis included 21 studies. The combined interventions resulted in a larger benefit in the EFs of older adults than that of the controls. However, there is a lack of a superiority of the combined interventions as compared to the cognitive or physical intervention alone. This is different from previous studies $[59,60]$, but it is quite original and interesting. Besides hypothesizing a role of the different choice of outcome measures mentioned above, it could also be speculated that the combined intervention, which was both mentally and physically challenging, could have resulted in excessive stress, less engagement in home or community-based activities, or other changes that inhibited rather than promoted neural plasticity and cognitive benefits [47]. This speculation may also explain why long intervention duration and high intervention frequency did not produce a significant effect. In this respect, sequential combinations require more time on the intervention and may cause more physical and cognitive load than simultaneous combination.

Although the combined intervention strategy was not found to be superior to physical or cognitive intervention alone in the present meta-analysis, the results confirmed that either type of intervention can affect EFs in older adults. Older adults should keep active, either physically or mentally, to prevent cognitive aging. It should be noted that in most of the literature reports analyzed, the combined intervention group was compared with a control group, while only a few studies compared the differences between the combined interventions and cognitive or physical intervention alone. This limitation may be due to the fact that the latter research design requires a larger number of subjects, which makes it difficult to carry out the research. Furthermore, the sample size was too small (fewer than 20 participants in each group) in some studies, which may cause a low reliability of the results. This is the reason why we preformed the current meta-analysis.

\section{Conclusions}

The combined physical and cognitive interventions effectively alleviated the decline of EFs in older adults. The effect of the combined interventions was affected by the length and frequency of the intervention as well as the quality of the research. However, based on the existing literature data, there 
is not enough evidence to prove that the combined intervention strategy has more advantages than physical exercise or cognitive intervention alone.

Supplementary Materials: The following are available online at http://www.mdpi.com/1660-4601/17/17/6166/s1, Table S1: Delphi list.

Author Contributions: Conceptualization, W.G. and B.W.; methodology, B.W.; formal analysis, M.Z. and S.K.; data curation, M.Z.; writing-Original draft preparation, W.G. and M.Z.; writing-Review and editing, S.K., M.S. and A.K.; visualization, S.K., M.S. and A.K.; supervision, B.W. All authors have read and agreed to the published version of the manuscript.

Funding: This research was funded by Natural Science Foundation of Jiangsu Province, grant number BK20180926, and Natural Science Foundation of Higher Education of Jiangsu Province, grant number 18KJD190004.

Acknowledgments: We are grateful for cooperation with Yangzhou University and Wrocław University School of Physical Education joint laboratory on sport and brain science.

Conflicts of Interest: The authors declare no conflict of interest.

\section{References}

1. Diamond, A. Executive functions. Ann. Rev. Psychol. 2013, 64, 135-168. [CrossRef] [PubMed]

2. Baddeley, A. Working Memory: Theories, Models, and Controversies. Ann. Rev. Psychol. 2012, 63, 1-29. [CrossRef] [PubMed]

3. Simpson, A.; Riggs, K.J.; Beck, S.R.; Gorniak, S.L.; Wu, Y.; Abbott, D.; Diamond, A. Refining the understanding of inhibitory processes: How response prepotency is created and overcome. Dev. Sci. 2011, 15, 62-73. [CrossRef]

4. Miyake, A.; Friedman, N.P.; Emerson, M.J.; Witzki, A.H.; Howerter, A.; Wager, T.D. The Unity and Diversity of Executive Functions and Their Contributions to Complex "Frontal Lobe" Tasks: A Latent Variable Analysis. Cogn. Psychol. 2000, 41, 49-100. [CrossRef] [PubMed]

5. Miyake, A.; Friedman, N.P. The Nature and Organization of Individual Differences in Executive Functions: Four General Conclusions. Curr. Dir. Psychol. Sci. 2012, 21, 8-14. [CrossRef]

6. Macpherson, S.E.; Phillips, L.H.; Della, S.S. Age, executive function, and social decision making: A dorsolateral prefrontal theory of cognitive aging. Psychol. Aging 2002, 17, 598-609. [CrossRef]

7. Elderkin-Thompson, V.; Ballmaier, M.; Hellemann, G.; Pham, D.; Kumar, A. Executive function and MRI prefrontal volumes among healthy older adults. Neuropsychology 2008, 22, 626-637. [CrossRef]

8. Abe, T.; Fujii, K.; Hyodo, K.; Kitano, N.; Okura, T. Effects of acute exercise in the sitting position on executive function evaluated by the Stroop task in healthy older adults. J. Phys. Ther. Sci. 2018, 30, 609-613. [CrossRef]

9. Carlson, M.C.; Saczynski, J.S.; Rebok, G.W.; Seeman, T.; Glass, T.A.; McGill, S.; Tielsch, J.M.; Frick, K.D.; Hill, J.; Fried, L.P. Exploring the effects of an "everyday" activity program on executive function and memory in older adults: Experience Corps. Gerontology 2008, 48, 793-801. [CrossRef]

10. Bixby, W.R.; Spalding, T.W.; Haufler, A.J.; Deeny, S.P.; Mahlow, P.T.; Zimmerman, J.B.; Hatfield, B.D. The Unique Relation of Physical Activity to Executive Function in Older Men and Women. Med. Sci. Sports Exerc. 2007, 39, 1408-1416. [CrossRef]

11. Best, J.R.; Nagamatsu, L.S.; Liu—Ambrose, T. Improvements to executive function during exercise training predict maintenance of physical activity over the following year. Front. Hum. Neurosci. 2014, 8, 353-362. [CrossRef] [PubMed]

12. Öhman, H.; Savikko, N.; Strandberg, T.E.; Pitkälä, K.H. Effect of Physical Exercise on Cognitive Performance in Older Adults with Mild Cognitive Impairment or Dementia: A Systematic Review. Dement. Geriatr. Cogn. Disord. 2014, 38, 347-365. [CrossRef] [PubMed]

13. Toril, P.; Reales, J.M.; Mayas, J.; Ballesteros, S. Video Game Training Enhances Visuospatial Working Memory and Episodic Memory in Older Adults. Front. Hum. Neurosci. 2016, 10, 206-219. [CrossRef] [PubMed]

14. Toril, P.; Reales, J.M.; Ballesteros, S. Video game training enhances cognition of older adults: A meta-analytic study. Psychol. Aging 2014, 29, 706-716. [CrossRef] [PubMed]

15. Shatil, E.; Mikulecka, J.; Bellotti, F.; Bureš, V. Novel Television-Based Cognitive Training Improves Working Memory and Executive Function. PLoS ONE 2014, 9, e101472. [CrossRef]

16. Haimov, I.; Shatil, E. Cognitive training improves sleep quality and cognitive function among older adults with insomnia. PLoS ONE 2013, 8, e61390. [CrossRef] 
17. Kim, M.-Y.; Kang, J.-W.; Park, S.-M.; Park, H.Y.; Park, J.-H. The Effect of Computer-Assisted Cognitive Training for Improving Cognitive Function in Older Adults: A Systematic Review. J. Korean Soc. Occup. Ther. 2016, 24, 81-94. [CrossRef]

18. Chu-Man, L.; Chang, M.-Y.; Chu, M.-C. Effects of mahjong on the cognitive function of middle-aged and older people. Int. J. Geriatr. Psychiatry 2015, 30, 995-997. [CrossRef]

19. Panphunpho, S.; Thavichachart, N.; Kritpet, T. Positive effects of Ska game practice on cognitive function among older adults. J. Med Assoc. Thail. Chotmaihet Thangphaet 2013, 96, 358-364.

20. Zhang, F.; Kaufman, D. Cognitive benefits of older adults' digital gameplay: A critical review. Gerontechnology 2016, 15, 3-16. [CrossRef]

21. Youn, J.H.; Park, S.; Lee, J.Y.; Cho, S.-J.; Kim, J.; Ryu, S.-H. Cognitive Improvement in Older Adults with Mild Cognitive Impairment: Evidence from a Multi-Strategic Metamemory Training. J. Clin. Med. 2020, 9, 362. [CrossRef] [PubMed]

22. Liu, X.Y.; Li, L.; Xiao, J.Q.; He, C.; Lyu, X.L.; Gao, L.; Yang, X.W.; Cui, X.G.; Fan, L.H. Cognitive Training in Older Adults with Mild Cognitive Impairment. Biomed. Environ. Sci. 2016, 29, 356-364. [PubMed]

23. Hill, N.T.M.; Mowszowski, L.; Naismith, S.L.; Chadwick, V.L.; Valenzuela, M.; Lampit, A. Computerized Cognitive Training in Older Adults with Mild Cognitive Impairment or Dementia: A Systematic Review and Meta-Analysis. Am. J. Psychiatry 2017, 174, 329-340. [CrossRef] [PubMed]

24. Eggenberger, P.; Schumacher, V.; Angst, M.; Theill, N.; de Bruin, E.D. Does multicomponent physical exercise with simultaneous cognitive training boost cognitive performance in older adults? A 6-month randomized controlled trial with a 1-year follow-up. Clin. Interv. Aging 2015, 10, 1335-1349. [CrossRef]

25. Kalbe, E.; Roheger, M.; Paluszak, K.; Meyer, J.; Becker, J.; Fink, G.R.; Kukolja, J.; Rahn, A.; Szabados, F.; Wirth, B.; et al. Effects of a Cognitive Training With and Without Additional Physical Activity in Healthy Older Adults: A Follow-Up 1 Year After a Randomized Controlled Trial. Front. Aging Neurosci. 2018, 10, 407-420. [CrossRef]

26. Barcelos, N.; Shah, N.; Cohen, K.; Hogan, M.J.; Mulkerrin, E.; Arciero, P.; Cohen, B.D.; Kramer, A.F.; Anderson-Hanley, C. Aerobic and Cognitive Exercise (ACE) Pilot Study for Older Adults: Executive Function Improves with Cognitive Challenge While Exergaming. J. Int. Neuropsychol. Soc. 2015, 21, 768-779. [CrossRef]

27. Okamura, H.; Otani, M.; Shimoyama, N.; Fujii, T. Combined Exercise and Cognitive Training System for Dementia Patients: A Randomized Controlled Trial. Dement. Geriatr. Cogn. Disord. 2018, 45, 318-325. [CrossRef]

28. Curlik, D.; Shors, T.J. Training your brain: Do mental and physical (MAP) training enhance cognition through the process of neurogenesis in the hippocampus? Neuropharmacology 2013, 64, 506-514. [CrossRef]

29. Fissler, P.; Küster, O.; Schlee, W.; Kolassa, I.-T. Novelty Interventions to Enhance Broad Cognitive Abilities and Prevent Dementia: Synergistic approaches for the facilitation of positive plastic change. Prog. Brain Res. 2013, 207, 403-434. [CrossRef]

30. Barban, F.; Annicchiarico, R.; Melideo, M.; Federici, A.; Lombardi, M.G.; Giuli, S.; Ricci, C.; Adriano, F.; Griffini, I.; Silvestri, M.; et al. Reducing Fall Risk with Combined Motor and Cognitive Training in Elderly Fallers. Brain Sci. 2017, 7, 19. [CrossRef]

31. Law, L.L.; Barnett, F.; Yau, M.K.; Gray, M. Effects of combined cognitive and exercise interventions on cognition in older adults with and without cognitive impairment: A systematic review. Ageing Res. Rev. 2014, 15, 61-75. [CrossRef]

32. Donnezan, L.C.; Perrot, A.; Belleville, S.; Bloch, F.; Kemoun, G. Effects of simultaneous aerobic and cognitive training on executive functions, cardiovascular fitness and functional abilities in older adults with mild cognitive impairment. Ment. Heal. Phys. Act. 2018, 15, 78-87. [CrossRef]

33. Bo, W.; Lei, M.; Tao, S.; Jie, L.T.; Qian, L.; Lin, F.Q.; Ping, W.X. Effects of combined intervention of physical exercise and cognitive training on cognitive function in stroke survivors with vascular cognitive impairment: A randomized controlled trial. Clin. Rehabil. 2018, 33, 54-63. [CrossRef] [PubMed]

34. Legault, C.; Jennings, J.M.; Katula, J.A.; Dagenbach, D.; Gaussoin, S.A.; Sink, K.M.; Rapp, S.R.; Rejeski, W.J.; Shumaker, S.A.; A Espeland, M. Designing clinical trials for assessing the effects of cognitive training and physical activity interventions on cognitive outcomes: The Seniors Health and Activity Research Program Pilot (SHARP-P) Study, a randomized controlled trial. BMC Geriatr. 2011, 11, 27. [CrossRef] [PubMed] 
35. Fossati, M.; Amati, F.; Painot, D.; Reiner, M.; Haenni, C.; Golay, A. Cognitive-behavioral therapy with simultaneous nutritional and physical activity education in obese patients with binge eating disorder. Eat. Weight. Disord. Stud. Anorexia Bulim. Obes. 2004, 9, 134-138. [CrossRef] [PubMed]

36. Laatar, R.; Kachouri, H.; Borji, R.; Rebai, H.; Sahli, S. Combined physical-cognitive training enhances postural performances during daily life tasks in older adults. Exp. Gerontol. 2018, 107, 91-97. [CrossRef]

37. Knaepen, K.; Goekint, M.; Heyman, E.; Meeusen, R. Neuroplasticity-Exercise-Induced Response of Peripheral Brain-Derived Neurotrophic Factor: A systematic review of experimental studies in human subjects. Sports Med. 2010, 40, 765-801. [CrossRef]

38. Berryman, C.; Stanton, T.; Bowering, K.J.; Tabor, A.; McFarlane, A.; Moseley, G.L.; Moseley, G. Do people with chronic pain have impaired executive function? A meta-analytical review. Clin. Psychol. Rev. 2014, 34, 563-579. [CrossRef]

39. Rahe, J.; Becker, J.; Fink, G.R.; Kessler, J.; Kukolja, J.; Rahn, A.; Rosen, J.B.; Szabados, F.; Wirth, B.; Kalbe, E. Cognitive training with and without additional physical activity in healthy older adults: Cognitive effects, neurobiological mechanisms, and prediction of training success. Front. Aging Neurosci. 2015, 7, 187-201. [CrossRef] [PubMed]

40. Verhagen, A.P.; de Vet, H.C.; de Bie, R.A.; Kessels, A.G.; Boers, M.; Bouter, L.M.; Knipschild, P.G. The Delphi list: A criteria list for quality assessment of randomized clinical trials for conducting systematic reviews developed by Delphi consensus. J. Clin. Epidemiol. 1998, 51, 1235-1241. [CrossRef]

41. Kayama, H.; Okamoto, K.; Nishiguchi, S.; Yamada, M.; Kuroda, T.; Aoyama, T.; Penichet, V.; Wolbring, G. Effect of a Kinect-Based Exercise Game on Improving Executive Cognitive Performance in Community-Dwelling Elderly: Case Control Study. J. Med. Internet Res. 2014, 16, e61. [CrossRef] [PubMed]

42. Schoene, D.; Lord, S.R.; Delbaere, K.; Severino, C.; Davies, T.A.; Smith, S.T. A Randomized Controlled Pilot Study of Home-Based Step Training in Older People Using Videogame Technology. PLoS ONE 2013, 8, e57734. [CrossRef] [PubMed]

43. Hiyamizu, M.; Morioka, S.; Shomoto, K.; Shimada, T. Effects of dual task balance training on dual task performance in elderly people: A randomized controlled trial. Clin. Rehabil. 2011, 26, 58-67. [CrossRef] [PubMed]

44. Shatil, E. Does combined cognitive training and physical activity training enhance cognitive abilities more than either alone? A four-condition randomized controlled trial among healthy older adults. Front. Aging Neurosci. 2013, 5, 1-12. [CrossRef]

45. Lam, L.; Chau, R.C.; Wong, B.M.; Fung, A.W.T.; Tam, C.W.; Leung, G.T.Y.; Kwok, T.C.; Leung, T.Y.; Ng, S.; Chan, W.M. A 1-Year Randomized Controlled Trial Comparing Mind Body Exercise (Tai Chi) With Stretching and Toning Exercise on Cognitive Function in Older Chinese Adults at Risk of Cognitive Decline. J. Am. Med Dir. Assoc. 2012, 13, 568.e15-568.e20. [CrossRef]

46. Sungkarat, S.; Boripuntakul, S.; Chattipakorn, N.; Watcharasaksilp, K.; Lord, S.R. Effects of Tai Chi on Cognition and Fall Risk in Older Adults with Mild Cognitive Impairment: A Randomized Controlled Trial. J. Am. Geriatr. Soc. 2016, 65, 721-727. [CrossRef]

47. Singh, M.A.F.; Gates, N.J.; Saigal, N.; Wilson, G.C.; Meiklejohn, J.; Brodaty, H.; Wen, W.; Singh, M.A.F.; Baune, B.T.; Suo, C.; et al. The Study of Mental and Resistance Training (SMART) Study-Resistance Training and/or Cognitive Training in Mild Cognitive Impairment: A Randomized, Double-Blind, Double-Sham Controlled Trial. J. Am. Med. Dir. Assoc. 2014, 15, 873-880. [CrossRef]

48. Park-Na, H.R.; Hiroyuki, S.; Kim-Jung, M.K. Combined Intervention of Physical Activity, Aerobic Exercise, and Cognitive Exercise Intervention to Prevent Cognitive Decline for Patients with Mild Cognitive Impairment: A Randomized Controlled Clinical Study. J. Clin. Med. 2019, 8, 940. [CrossRef]

49. Maillot, P.; Perrot, A.; Hartley, A. Effects of interactive physical-activity video-game training on physical and cognitive function in older adults. Psychol. Aging 2012, 27, 589-600. [CrossRef]

50. Barnes, D.E.; Santos-Modesitt, W.; Poelke, G.; Kramer, A.F.; Castro, C.; Middleton, L.E.; Yaffe, K. The mental activity and exercise (MAX) trial a randomized controlled trial to enhance cognitive function in older adults. JAMA Intern. Med. 2013, 173, 797-804. [CrossRef]

51. Mrakic-Sposta, S.; di Santo, S.G.; Franchini, F.; Arlati, S.; Zangiacomi, A.; Greci, L.; Moretti, S.; Jesuthasan, N.; Marzorati, M.; Rizzo, G.; et al. Effects of Combined Physical and Cognitive Virtual Reality-Based Training on Cognitive Impairment and Oxidative Stress in MCI Patients: A Pilot Study. Front. Aging Neurosci. 2018, 10, 282-292. [CrossRef] [PubMed] 
52. Brinke, L.F.T.; Best, J.R.; Chan, J.L.C.; Ghag, C.; Erickson, K.I.; Handy, T.C.; Liu, A.T. The Effects of Computerized Cognitive Training with and without Physical Exercise on Cognitive Function in Older Adults: An 8-week Randomized Controlled Trial. J. Gerontol. Ser. A Boil. Sci. Med. Sci. 2019, 75, 755-763. [CrossRef]

53. Shah, T.M.; Verdile, G.; Sohrabi, H.R.; Campbell, A.; Putland, E.; Cheetham, C.; Dhaliwal, S.; Weinborn, M.; Maruff, P.; Darby, D.; et al. A combination of physical activity and computerized brain training improves verbal memory and increases cerebral glucose metabolism in the elderly. Transl. Psychiatry 2014, 4, e487. [CrossRef] [PubMed]

54. Bae, S.; Lee, S.; Jung, S.; Makino, K.; Harada, K.; Harada, K.; Shinkai, Y.; Chiba, I.; Shimada, H. The effect of a multicomponent intervention to promote community activity on cognitive function in older adults with mild cognitive impairment: A randomized controlled trial. Complement. Ther. Med. 2019, 42, 164-169. [CrossRef]

55. Nishiguchi, S.; Yamada, M.; Tanigawa, T.; Sekiyama, K.; Kawagoe, T.; Suzuki, M.; Yoshikawa, S.; Abe, N.; Otsuka, Y.; Nakai, R.; et al. A 12-Week Physical and Cognitive Exercise Program Can Improve Cognitive Function and Neural Efficiency in Community-Dwelling Older Adults: A Randomized Controlled Trial. J. Am. Geriatr. Soc. 2015, 63, 1355-1363. [CrossRef] [PubMed]

56. Linde, K.; Alfermann, D. Single versus combined cognitive and physical activity effects on fluid cognitive abilities of healthy older adults: A 4-month randomized controlled trial with follow-up. J. Aging Phys. Act. 2014, 22, 302-313. [CrossRef] [PubMed]

57. Rahe, J.; Petrelli, A.; Kaesberg, S.; Fink, G.R.; Kessler, J.; Kalbe, E. Effects of cognitive training with additional physical activity compared to pure cognitive training in healthy older adults. Clin. Interv. Aging 10, 297-310. [CrossRef]

58. Hagovska, M.; Nagyova, I. The transfer of skills from cognitive and physical training to activities of daily living: A randomised controlled study. Eur. J. Ageing 2016, 14, 133-142. [CrossRef]

59. Zhu, X.; Yin, S.; Lang, M.; He, R.; Li, J. The more the better? A meta-analysis on effects of combined cognitive and physical intervention on cognition in healthy older adults. Ageing Res. Rev. 2016, 31, 67-79. [CrossRef]

60. Gheysen, F.; Poppe, L.; Desmet, A.; Swinnen, S.; Cardon, G.; de Bourdeaudhuij, I.; Chastin, S.; Fias, W. Physical activity to improve cognition in older adults: Can physical activity programs enriched with cognitive challenges enhance the effects? A systematic review and meta-analysis. Int. J. Behav. Nutr. Phys. Act. 2018, 15, 63. [CrossRef]

61. Walsh, J.N.; Manor, B.; Hausdorff, J.; Novak, V.; Lipsitz, L.; Gow, B.; Macklin, E.A.; Peng, C.-K.; Wayne, P.M. Impact of Short- and Long-term Tai Chi Mind-Body Exercise Training on Cognitive Function in Healthy Adults: Results from a Hybrid Observational Study and Randomized Trial. Glob. Adv. Heal. Med. 2015, 4, 38-48. [CrossRef]

62. Holtzer, R.; Shuman, M.; Mahoney, J.R.; Lipton, R.; Verghese, J. Cognitive Fatigue Defined in the Context of Attention Networks. Aging Neuropsychol. Cogn. 2010, 18, 108-128. [CrossRef]

63. Gutchess, A. Plasticity of the aging brain: New directions in cognitive neuroscience. Science 2014, 346, 579-582. [CrossRef]

64. Hedge, C.; Powell, G.; Sumner, P. The mapping between transformed reaction time costs and models of processing in aging and cognition. Psychol. Aging 2018, 33, 1093-1104. [CrossRef]

65. Rabbitt, P. Do "Frontal Tests" Measure Executive Function? Issues of Assessment and Evidence from Fluency Tests. In Methodology of Frontal and Executive Function; Routledge: London, UK, 2004; pp. 193-215.

66. Northey, J.M.; Cherbuin, N.; Pumpa, K.L.; Smee, D.J.; Rattray, B. Exercise interventions for cognitive function in adults older than 50: A systematic review with meta-analysis. Br. J. Sports Med. 2017, 52, 154-160. [CrossRef]

67. Kramer, A.F.; Colcombe, S.J. Fitness Effects on the Cognitive Function of Older Adults: A Meta-Analytic Study-Revisited. Perspect. Psychol. Sci. 2018, 13, 213-217. [CrossRef] 
68. Friedman, N.P.; Miyake, A.; Altamirano, L.J.; Corley, R.P.; Young, S.E.; Rhea, S.A.; Hewitt, J.K. Stability and change in executive function abilities from late adolescence to early adulthood: A longitudinal twin study. Dev. Psychol. 2016, 52, 326-340. [CrossRef]

69. McEwen, S.C.; Siddarth, P.; Abedelsater, B.; Kim, Y.; Mui, W.; Wu, P.; Emerson, N.D.; Lee, J.; Greenberg, S.; Shelton, T.; et al. Simultaneous Aerobic Exercise and Memory Training Program in Older Adults with Subjective Memory Impairments. J. Alzheimer's Dis. 2018, 62, 795-806. [CrossRef]

(C) 2020 by the authors. Licensee MDPI, Basel, Switzerland. This article is an open access article distributed under the terms and conditions of the Creative Commons Attribution (CC BY) license (http://creativecommons.org/licenses/by/4.0/). 